\title{
Evaluating bowel enterotomy closures in simulated deep body cavities using the reversing half-hitch alternating post and square knots: a randomized controlled trial
}

\author{
Edward A. Sykes, MD, PhD \\ Madeline Lemke, MD, MSc \\ Daniel Potter, MD \\ Terry Li, PhD \\ Zuhaib M. Mir, MD, MSc \\ Guy Sheahan, MBBS, MEd \\ Vincent $\mathrm{Wu}, \mathrm{MD}$ \\ Boris Zevin, MD, PhD
}

This work was presented as a podium presentation at the American College of Surgeons Surgical Simulation Summit, Chicago, Illinois, March 2019, and as a poster at the Canadian Surgery Forum, Montreal, Québec, September 2019.

Accepted March 16, 2020

\section{Correspondence to: \\ Boris Zevin MD, PhD \\ Department of Surgery \\ Kingston General Hospital \\ 76 Stuart Street \\ Kingston, ON K7L 2V7 \\ boris.zevin@kingstonhsc.ca}

DOI: $10.1503 /$ cjs.016719

\begin{abstract}
Background: Square knots can be difficult to construct in deep body cavities. The reversing half-hitch alternating post (RHAP) surgical knot has noninferior tensile strength and performance characteristics in deep body cavities. We compared the enterotomy repairs of novice learners in simulated deep body cavities using RHAP versus square knots after proficiency-based training.
\end{abstract}

Methods: Undergraduate students were randomized to RHAP $(n=10)$ or square knot $(n=10)$ groups and trained to defined proficiency. They then performed hand-sewn enterotomy repairs of cadaveric porcine small bowels on flat surfaces and in simulated deep body cavities. We recorded time to knot-tying proficiency and to enterotomy repair, and burst pressures for the repair.

Results: Mean time-to-proficiency in knot tying was equivalent between the RHAP and square knot groups (23 [standard deviation (SD) 3] v. 21 [SD 2] min, $p=0.33$ ). Mean time for enterotomy repair in deep cavities was shorter for the RHAP group (16 [SD 2] min v. 21 [SD 1] $\min , p=0.02$ ). Mean burst pressures for enterotomy repair were equivalent on flat surfaces $(128$ [SD 41] v. $101[\mathrm{SD} 36] \mathrm{mm} \mathrm{Hg}, p=0.31)$, and were significantly higher for the RHAP group in simulated deep body cavities (32 [SD 13] v. 105 [SD 37] $\mathrm{mm} \mathrm{Hg}, p=0.05$ ).

Conclusion: The RHAP knots appear to have superior performance versus square knots when tied in a deep body cavity by novice learners. Future work should focus on demonstrating the clinical relevance and broad utility of the RHAP knot in abdominal surgery. Both knot types should be taught to novice learners.

Contexte : L'exécution de nœuds plats peut être difficile dans les cavités corporelles profondes. Les nœuds de type demi-clé inversée alternée (RHAP, pour reversing balfbitch alternating post) ont une résistance à la traction et un rendement semblables à ceux des nœuds plats dans ces cavités. Nous avons comparé l'efficacité des nœuds plats et des nœuds de type RHAP réalisés par de nouveaux apprenants dans des cavités profondes simulées, après leur avoir enseigné les compétences nécessaires.

Méthodes : Les étudiants de premier cycle ont été aléatoirement répartis en 2 groupes, soit le groupe $\operatorname{RHAP}(n=10)$ et le groupe noud plat $(n=10)$, et ont reçu une formation pour développer des compétences prédéfinies. Ils ont ensuite suturé à la main un intestin grêle provenant d'un cadavre de porc, sur une surface plane et à l'intérieur d'une cavité profonde simulée. Nous avons mesuré le temps nécessaire à l'exécution du noud et à la suture complète de l'incision, de même que la pression que pouvait subir cette suture sans se rompre.

Résultats : Le temps moyen d'exécution du noud était semblable entre les groupes RHAP et nœud plat (23 min [écart type (E.T.) $3 \mathrm{~min}$ ] c. $21 \mathrm{~min}$ [E.T. $2 \mathrm{~min}$ ]; $p=0,33$ ). Le temps moyen nécessaire à la suture de l'incision dans la cavité profonde était plus court dans le groupe RHAP (16 $\mathrm{min}$ [E.T. $2 \mathrm{~min}$ ] c. $21 \mathrm{~min}$ [E.T. $1 \mathrm{~min}$; $p=0,02)$. La pression moyenne que pouvait subir la suture sans se rompre était comparable pour les sutures effectuées sur une surface plane $(128 \mathrm{~mm} \mathrm{Hg}$ [E.T. $41 \mathrm{~mm} \mathrm{Hg}$ c. $101 \mathrm{~mm} \mathrm{Hg}$ [E.T. $36 \mathrm{~mm} \mathrm{Hg}$; $p=0,31$ ), mais était significativement plus élevée dans le groupe RHAP pour les sutures faites dans la cavité profonde $(32 \mathrm{~mm} \mathrm{Hg}$ [E.T. $13 \mathrm{~mm} \mathrm{Hg}$ ] c. $105 \mathrm{~mm} \mathrm{Hg}$ [E.T. $37 \mathrm{~mm} \mathrm{Hg}$, $p=0,05)$.

Conclusion : Les nœuds de type RHAP semblent avoir un rendement supérieur à celui des nœuds plats lorsqu'ils sont réalisés dans une cavité profonde par de nouveaux apprenants. Des études ultérieures devraient se pencher sur la pertinence clinique et l'utilité générale de ces nœuds en chirurgie abdominale. Les 2 types de nœuds devraient être enseignés aux nouveaux apprenants. 


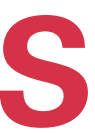

uturing and hand-tying surgical knots are essential skills for a variety of medical specialties. Various surgical knot configurations with different tensile strengths and mechanisms of failure have been developed for different applications. ${ }^{1}$ Despite the variety, the square knot remains the surgical gold standard. Composed of alternating throws that are stacked in parallel, the square knot allows suture strands to self-tighten under tension and maximize frictional surface area and knot security (Figure 1A). ${ }^{2}$ Unfortunately, the construction of square knots requires an accessible working area to ensure that tension is applied perpendicular and lateral to the wound so that each throw is laid flat on top of the previous. Failure to do so leads to the formation of a "slip knot," which has inferior tensile properties. This technical limitation makes square knots difficult to perform for surgeries within deep confined spaces, which may inadvertently result in the production of surgical slip knots. ${ }^{3,4}$ The reversing half-hitch alternating post (RHAP) is an alternative to the square knot, created initially for arthroscopic procedures. ${ }^{5}$ This knot is produced by first laying a slip knot followed by successive hitch knots, where the post is alternated between each throw (Figure 1B). ${ }^{3}$ This allows hitches to be tied at a distance and translated along the post to the closure site. As such, this knot requires less working space to construct. ${ }^{3,4,6}$ Step-by-step details on how to construct this knot can be found in our previous publications. ${ }^{6,7}$ We have previously shown that the RHAP knot exhibits equivalent tensile strength to square knots. ${ }^{3,6} \mathrm{We}$ have also shown that RHAP knots are easier for medical students to learn and to tie at depth than square knots in a simulated setting. ${ }^{7}$ These capabilities suggest that this knot-tying technique may be useful for learners in surgery to perform while perfecting new surgical techniques. The next step toward generalizability of the RHAP knot in medical education is to assess the quality of this knot in more clinically relevant scenarios. One such scenario is the repair of a small bowel enterotomy, where the difficulty of simultaneously ensuring proper repair and operating within a deep body cavity may be hard to balance. However, the utility of the RHAP knot for repair of enterotomies in deep body cavities is yet to be established. Hence, the purpose of this study was to compare the use of RHAP and square knots to repair small bowel enterotomies in a simulated deep body cavity following proficiency-based training in knot tying for novice learners.

\section{Methods}

We conducted a prospective, single-blinded, two-arm, parallel randomized controlled trial with adult undergraduate students from Queen's University. We randomized participants to either the square knot (control) or the RHAP knot (intervention) groups. All results are reported in adherence with the CONSORT 2010 statement. ${ }^{8}$ This

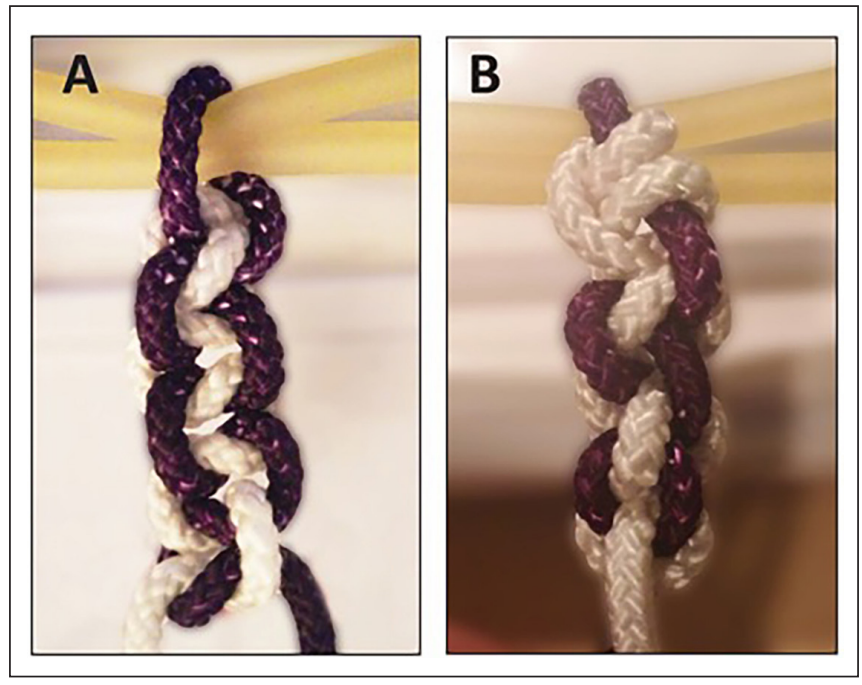

Fig. 1. Pictorial representation of $(A)$ square and $(B)$ reverse halfhitch alternating post knots. Photographs taken by Edward Sykes.

study was registered with ClinicalTrials.gov, identifier NCT03742583. All testing occurred at Queen's University in the Surgical Skills Simulation Laboratory. The study was approved by the Queen's University Health Sciences and Affiliated Teaching Hospitals Research Ethics Board and Queen's University Undergraduate Medical Education Curriculum Committee.

\section{Participants}

We recruited undergraduate students from Queen's University who were older than 18 years of age and currently enrolled in an undergraduate program (nonmedical or preclerkship medical school). We excluded participants who had begun clerkship/hospital training or who had completed an undergraduate medical program because of their pre-existing experience in surgery. This ensured that knot-tying and prior surgical experience for both groups were balanced, and simulated a real-world scenario where a learner is not familiar with a new surgical technique. We recruited participants via email and obtained written consent from all participants before beginning the study. Randomization of group allocation was achieved through random number generation from Microsoft Excel 2016, performed by study personnel. Neither participants nor instructors were blinded to the group allocation; however, assessment of burst pressure (primary outcome) was blinded to group allocation.

\section{Sample size}

We calculated a minimum sample size of 8 participants per group based on a superiority study design, with a power of $80 \%$ and an $\alpha$ of 0.05 , established based on a superior burst pressure of $75 \%$ or greater. Mean burst pressures were obtained from previous research examining 
medical students performing porcine bowel anastomoses; means of baseline scores were used (1.02 [standard deviation (SD) 0.56] $\mathrm{mm} \mathrm{Hg}$. ${ }^{\text {? }}$

\section{Training and assessment}

Participants were assessed at baseline for their ability to hand tie their assigned surgical knot (square or RHAP) using a visual aid (Figure 1). Nonblinded assessors scored each participant on their knot-tying technique using a previously published and validated knot-tying checklist. ${ }^{10}$ Proficiency in knot-tying for this study was defined as being able to perform 5 out of 6 of the following criteria: securing a post, tying the knot at a correct distance, appropriately grasping suture ends, appropriately gathering the loose ends without dropping the suture, appropriately laying down a throw and completing 4 throws without an air knot. ${ }^{10}$ The same criteria for knot-tying proficiency was used for both square and RHAP knots. Correct reproduction of the assigned knot was required in order to be deemed proficient. Participants were coached and were provided time to individually practise their knot tying on surgical knot-tying boards (Ethicon) until defined proficiency was attained. The time required to achieve proficiency was recorded for each participant.

\section{Repair of enterotomy in a synthetic model}

Participants who demonstrated proficiency in knot tying were individually instructed how to perform small bowel enterotomy repairs using a synthetic model (Figure 2A). For consistency the same instructor provided instruction to

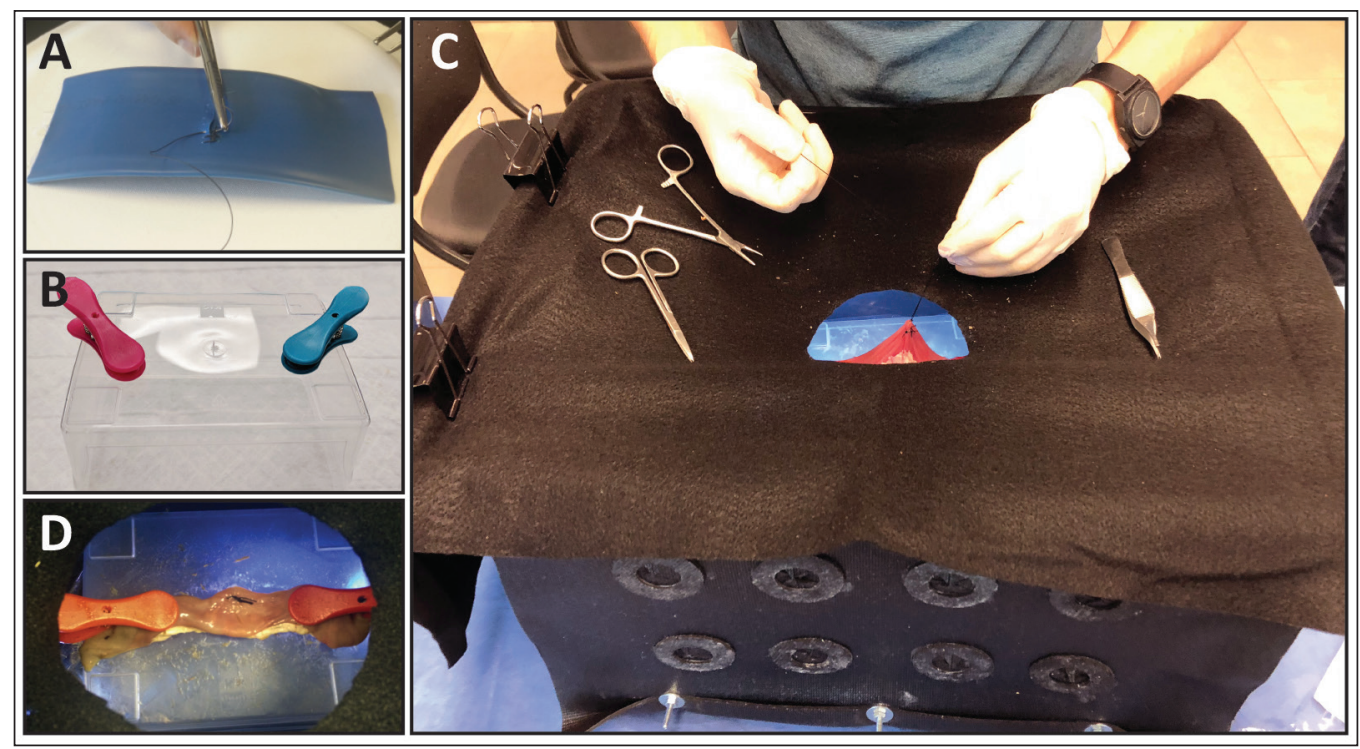

Fig. 2. Experimental set up of $(A)$ small bowel enterotomy model used for suture and surgical hand-tie training. (B) Platform used for flat surface enterotomy repair of cadaveric porcine small bowel. (C) Simulated deep body cavity used for enterotomy repair of cadaveric porcine small bowel. (D) Top-down view of surgical opening of the simulated deep body cavity. Images are not to scale. Photographs taken by Edward Sykes. all participants. Participants were coached on how to perform full thickness enterotomy repair by applying simple interrupted 2-0 gauged silk sutures (Ethicon), spaced $3 \mathrm{~mm}$ apart along the entire length of the enterotomy. Simulated models of enterotomy repair were constructed using a $10 \mathrm{~cm}$ length of flat PVC discharge hose (2 inch diameter, Eastman) with a single-wall transverse incision $(1-2 \mathrm{~cm})$ to mimic a small bowel enterotomy. Models were affixed to a board for stability. Participants were provided 30 minutes to practise the enterotomy repair, with individualized coaching to ensure proper suturing and repair technique.

\section{Repair of enterotomy in a cadaveric porcine small bowel model}

After training on a synthetic model, participants were instructed how to repair an enterotomy on a cadaveric porcine small bowel model. A $1 \mathrm{~cm}$ longitudinal enterotomy was made on the antimesenteric border of a fresh segment $(15 \mathrm{~cm})$ of cadaveric porcine small bowel, mounted on a plastic board with clips at both ends (Figure 2B). The length of enterotomy was selected to model the degree of iatrogenic bowel injury that might occur during surgery and to minimize the time required for novices to perform the enterotomy repair. Participants were instructed to close the enterotomy using simple interrupted $2-0$ silk sutures (Ethicon) with either RHAP or square knot hand ties, according to their group allocation. Each participant performed 1 enterotomy repair on a flat surface (Figure 2B) and another in a simulated deep body cavity (Figure 2C). We constructed simulated deep body cavities from clear tote boxes $(38 \times 25 \times 27 \mathrm{~cm})$ covered with black felt, containing a circular opening with a diameter of $10 \mathrm{~cm}$ (Figure 2D). Cadaveric porcine small bowel specimens were fixed onto the centre of the deep body cavity, $10 \mathrm{~cm}$ below the circular opening. We placed lights inside the box for illumination. After completion of the enterotomy repair, specimens were stored at room temperature in plastic bags labelled with the participant's identification number.

\section{Assessment of burst pressure for enterotomy repair}

We performed burst pressure testing on the same day as the 


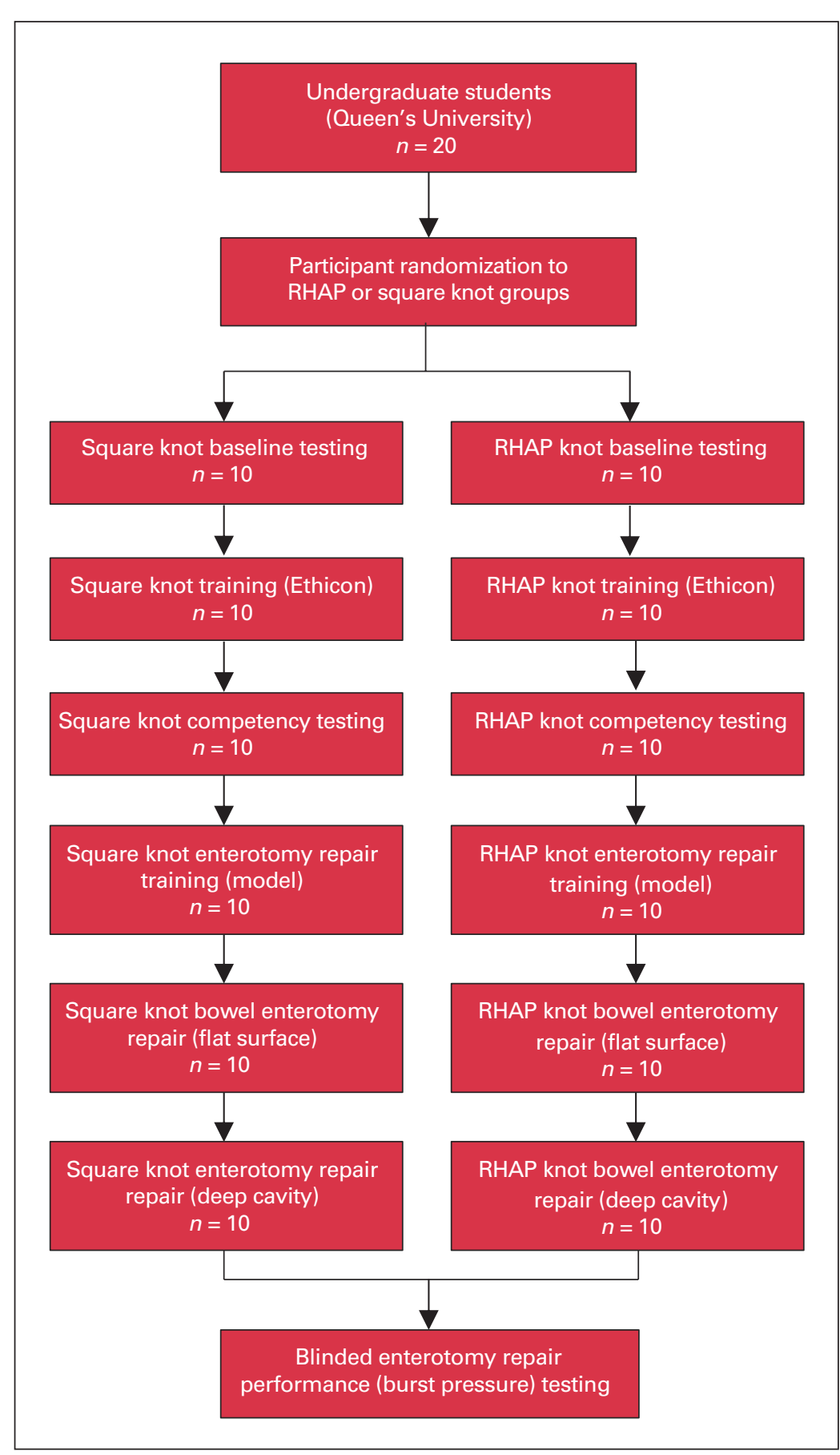

Fig. 3. Participant flow diagram. RHAP = reverse half-hitch alternating post. chloride), pre-mixed with blue food colouring to facilitate visualization of fluid on the white surface. The saline bag was hung $100 \mathrm{~cm}$ above the specimen and was wrapped with an aneroid sphygmomanometer (Tycos Classic DS48A) cuff. The specimen was then filled with the coloured saline via intravenous tubing under gravity. Once filled, the pressure cuff on the saline bag was slowly inflated to gradually increase the luminal hydrostatic pressure and carefully monitored for fluid leakage from the enterotomy repair site. The sphygmomanometer pressure at which the fluid leakage was first visualized was recorded as the burst pressure in $\mathrm{mm} \mathrm{Hg}$. Any saline leakage from the enterotomy site under gravity (before pressure was applied) was recorded as a score of $0 \mathrm{~mm} \mathrm{Hg}$. A value of $300 \mathrm{~mm} \mathrm{Hg}$ was assigned to all specimens that did not leak at pressures greater than $300 \mathrm{~mm} \mathrm{Hg}$, as this was the limit of the pressure gauge. Assessment of burst pressure was blinded to participant number and group allocation, but not the type of specimen (flat surface versus deep body cavity).

\section{Statistical analysis}

We performed simple descriptive statistics for time, knot-tying checklist scores and burst pressure measurements. We used Student $t$ tests for between-group comparisons of time to achieve proficiency in knot-tying and burst pressure. Error values reported in this document denote the standard error of the mean with $n=10$ for each group. Time measurements were rounded to the nearest whole number. GraphPad Prism (version 7.04, GraphPad Software) were used for all statistical analysis, with significance set to $p<0.05$.

\section{REsults}

Twenty participants (9 female and 11 male) were recruited and randomized equally into the square or RHAP knot-tying groups (Figure 3). At baseline, none of the study partici-

enterotomy repair. The quality of the enterotomy repair was assessed using the hydrostatic pressure necessary to cause leakage at the enterotomy site (burst pressure). We placed specimens in a white basin to facilitate visualization. We inserted intravenous tubing into the intestinal lumen proximal to the enterotomy site and sealed it tightly with 2-0 silk sutures, circumferentially. We sealed the distal end with a Kocher clamp. We then attached the intravenous tubing to a $1 \mathrm{~L}$ bag of normal saline $(0.9 \%$ sodium pants were proficient in knot tying. The mean times required for each participant to become proficient in knot tying for the RHAP (23 [SD 3] min) and square knots (21 [SD 2] min) were equivalent $(p=0.33)$ (Figure 4).

\section{Enterotomy repair}

There was no difference between the square knot and RHAP groups in time required to repair the cadaveric 


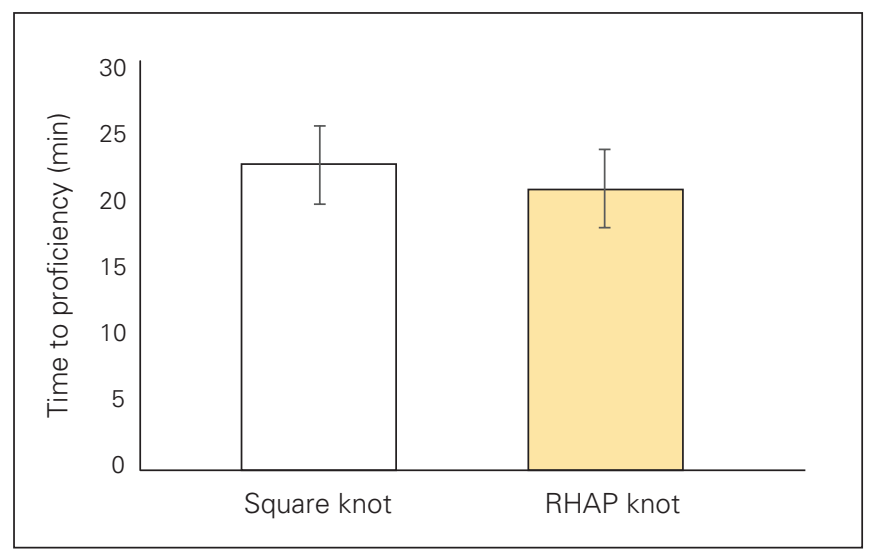

Fig. 4. Comparison of time to achieve proficiency in knot tying for square and reverse half-hitch alternating post (RHAP) knot groups. Error bars denote standard of error of mean $(n=10)$.

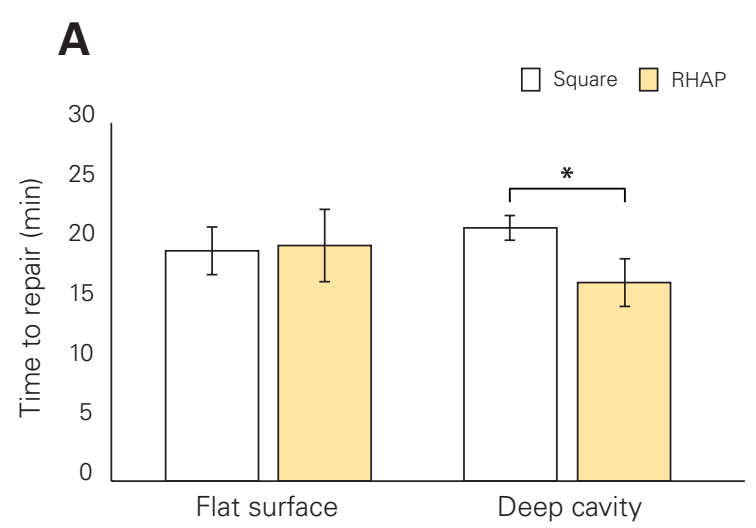

B

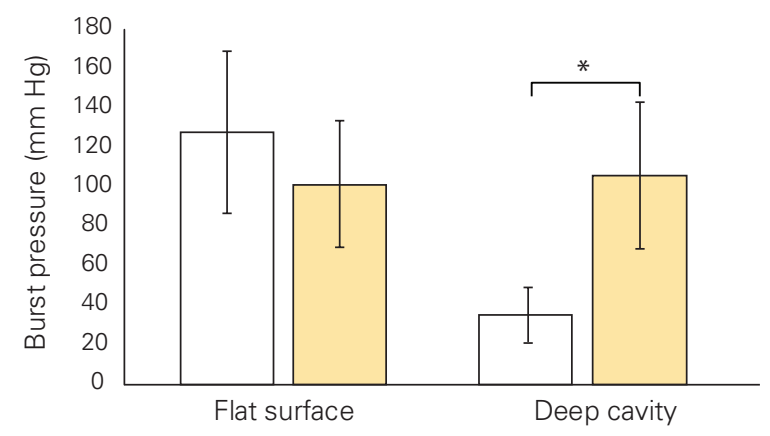

Fig. 5. Comparison of (A) time and (B) burst pressure for enterotomy repairs performed on a flat surface and in a simulated deep body cavity for square and reverse half-hitch alternating post (RHAP) knot groups. Error bars denote standard of error of mean $(n=10) .{ }^{*} p<0.05$.

porcine small bowel enterotomy on a flat surface (19 [SD 2] v. 19 [SD 2] min, $p=0.45$ ) (Figure 5A). Repairs performed by the RHAP knot group in simulated deep body cavities were significantly faster than repairs performed by the square knot group (16 [SD 2] v. 21 [SD 1] min, $p=0.04$ ) (Figure 5A). The mean burst pres- sures for enterotomy repairs on a flat surface were statistically similar for the square and RHAP knot groups (128 [SD 40] v. 101 [SD 35] mm Hg, $p=0.31$ ) (Figure 5B). In simulated deep body cavities, the burst pressure for enterotomy repairs using the square knot was significantly lower than that of the RHAP knot (35 [SD 14] v. 105 [SD 39] $\mathrm{mm} \mathrm{Hg}, p=0.05$ ) (Figure 5B). There was a statistically significant within-group difference in burst pressure for enterotomy repair using a square knot on a flat surface and a deep body cavity $(p=0.03)$. There was no statistically significant within-group difference between the 2 surfaces for enterotomy repairs using the RHAP $\operatorname{knot}(p=0.77)$.

\section{Discussion}

We conducted a prospective, single-blinded randomized controlled trial to compare the use of RHAP and square knots to repair small bowel enterotomies in a simulated deep body cavity following proficiency-based training in surgical knot-tying for novice learners. Using a graduated curriculum, we endeavoured to parallel the clinical experiences of novice learners who are proficient at knot tying and are now required to perform a repair of a small bowel enterotomy for the first time in a deep body cavity. We showed that novice learners can achieve proficiency in knot tying after 25 minutes of deliberate practice and structured feedback. We also showed that the integrity of enterotomy repairs performed on a flat surface - as measured by burst pressure causing fluid leakage at the repair site - was equivalent for RHAP and square knots. However, the integrity of enterotomy repairs performed in a simulated deep body cavity was significantly higher when repaired using the RHAP knot, suggesting that RHAP knots are more secure and easier to construct for novice learners in a deep body cavity compared with square knots. These findings are important to surgical educators, medical students and surgical trainees, as all practising general surgeons are required to repair enterotomies in their clinical practice. In this study, we developed a simulation-based curriculum that focused on the deliberate practice of surgical knot tying, suturing and small bowel enterotomy repairs as a means of enhancing surgical skills. It provided learners with specific goals and real-time feedback from preceptors using a previously validated rubric for surgical knot tying. Moreover, our low- and intermediate-fidelity bowel enterotomy models allowed learners to repetitively practise suture placement and knot tying on reusable synthetic models, while appreciating the tactile delicacy of soft tissues using cadaveric porcine small bowels. Known collectively as deliberate practice, the combination of peer feedback, self-reflection and a simulation platform provides a structured manner for learners to become self-aware of errors, gain experience on new surgical skills and improve performance within a 2 -hour training session. ${ }^{11,12}$ Our 
approach may be considerably faster than other training modalities, such as didactic surgical video coaching or resident-directed practice, as we incorporate salient features of both training schemes. ${ }^{13,14}$ However, successful implementation of this training curriculum requires increased learner supervision, experienced instructors and higher costs for simulated models than the other training programs. Enterotomy repair can be difficult to perform in deep body cavities using standard square surgical knots due to limitations in working space. This difficulty is further compounded in novice learners who have yet to master basic knot-tying technique.

Our results demonstrate that enterotomy repairs in simulated deep body cavities are faster to perform and more secure with the RHAP knot than with the square knot. We have previously shown that the RHAP knot is easier for novices to learn to tie at depth, ${ }^{7}$ and we now hypothesize that the reduced complexity of the RHAP knot allows the learner to focus their attention on repairing the enterotomy rather than on tying the knots. Our hypothesis is supported by the observed deterioration in performance (burst pressure) seen in the square knot group when participants transitioned from a flat surface to a simulated deep body cavity. We surmise that the RHAP knot provides better success in knot tying in volumetrically restricted spaces because it allows throws to be tied at a distance, translated to the ligature and secured with a single digit under tension. ${ }^{4}$ By virtue of this simplification, we hypothesize that the RHAP knot may reduce the likelihood of errors in knot tying during surgery.

Burst pressure, the hydrostatic pressure necessary to cause suture-line leakage, as used as a surrogate for the quality of enterotomy repair. ${ }^{15}$ The objective of our study was focused on comparing the real-world performance of enterotomy repairs completed by novice learners who are proficient in knot tying, rather than focusing on the proficiency-based training of enterotomy repairs. The statistically higher burst pressures in a deep body cavity seen in the RHAP group suggests that the RHAP knot may be advantageous when surgical space is limited and when people are unfamiliar with the surgical task. The mean burst pressures reported in our study are comparable to pressures reported in other studies. ${ }^{16,17}$ This supports the validity of our results and the possibility that, with future research, the RHAP knot may become an alternative to the square knot in clinically relevant scenarios. ${ }^{16-18}$ As such, we suggest teaching the RHAP knot to novice learners in addition to the square knot as it will allow novices to appropriately select their knot type according to the available working space, and to minimize failure rates of their knots.

\section{Limitations}

There are several limitations that restrict the generalizability of our findings. First, we examined the performance of novice learners and our results may not be generalizable to junior-, intermediate- and senior-level residents or experienced surgeons. Second, we conducted our study in a simulated environment, and although previous research has shown that surgical skills transfer from a simulated environment to the operating room, ${ }^{19}$ we did not confirm this in our study. Third, we did not perform microscopic examination of square knots and RHAP knots to identify loose or slipped knots on the enterotomy repair specimens because we wanted to mimic the setting of the operating room, where it may be difficult to assess the presence of loose or slipped knots. Our previous work ${ }^{7}$ showed no difference in the frequency of slipped RHAP versus square knots when constructed at depth. Fourth, we used braided nonabsorbable $2-0$ silk sutures for enterotomy repairs. The differences in burst pressures between the 2 knot types may not be generalizable to other suture materials that exhibit differing elastic and frictional properties, such as monofilament sutures. Fifth, we did not provide specific instructions on the number and bite width of simple interrupted sutures to repair the enterotomy. This could have resulted in variation between participants, but should have remained balanced between the 2 interventions. Sixth, we did not assess the process of repairing the enterotomy; only the quality of the final product was evaluated using burst pressure. We did not teach participants enterotomy repair to proficiency. This was intentionally done to compare how well surgical trainees who have been taught both knots can learn and complete procedures in the operating room using these knots. Lastly, the findings of our study are only representative of early technical failures in enterotomy repair. These results cannot be extrapolated to delayed failures associated with healing and patient factors such as malnutrition, immunosuppression and field contamination.

\section{Conclusion}

Novice learners were able to repair small bowel enterotomies in a simulated deep body cavity faster and more securely using RHAP knots versus square knots. We suggest incorporating RHAP knots into proficiency-based knot-tying curricula for novice learners, in addition to square knots, to allow trainees to appropriately select their knot type according to the available working space to minimize the failure rates of their knots.

Acknowledgements: The authors thank the Clinical Simulation Centre at Queen's University and its administrative staff for funding and access to their facility. The authors would also like to acknowledge Edbert Khong and Stella Tung for their aid in data acquisition.

Affiliations: From the School of Medicine, Queen's University, Kingston, Ont. (Sykes, Lemke, Potter, Zevin); the Department of Biomedical and Molecular Sciences, Queen's University, Kingston, Ont. (Li); the Department of Surgery, Division of General Surgery, Queen's University, Kingston, Ont. (Mir, Sheahan, Zevin); and the Department of Otolaryngology - Head and Neck Surgery, University of Toronto, Toronto, Ont. (Sykes, Wu). 


\section{Competing interests: None declared.}

Funding: This work was supported by the Queen's University Clinical Simulation Centre Summer Research Student Grant.

Contributors: E. Sykes, M. Lemke, V. Wu and B. Zevin designed the study. E. Sykes, M. Lemke, T. Li, D. Potter, G. Sheahan and Z. Mir acquired the data, which E. Sykes and M. Lemke analyzed. E. Sykes, M. Lemke, D. Potter and B. Zevin wrote the article, which all authors reviewed and approved for publication.

Content licence: This is an Open Access article distributed in accordance with the terms of the Creative Commons Attribution (CC BYNC-ND 4.0) licence, which permits use, distribution and reproduction in any medium, provided that the original publication is properly cited, the use is noncommercial (i.e., research or educational use), and no modifications or adaptations are made. See: https://creativecommons. org/licenses/by-nc-nd/4.0/

\section{References}

1. Silver E, Wu R, Grady J, et al. Knot security- how is it affected by suture technique, material, size, and number of throws? 7 Oral Maxillofac Surg 2016;74:1304-12.

2. Brown RP. Knotting technique and suture materials. Br 7 Surg 1992;79:399-400.

3. Gunderson PE. The half-hitch knot: a rational alternative to the square knot. Am 7 Surg 1987;154:538-40.

4. Chong ACM, Prohaska DJ, Pate RC. Reverse flipping technique: an alternate approach to tie reversing half-hitches on alternating posts. Artbrosc Tech 2016;5:e403-5.

5. Loutzenheiser TD, Harryman DT, Yung SW, et al. Optimizing arthroscopic knots. Artbroscopy 1995;11:199-206.

6. Wu V, Sykes EA, Mercer D, et al. Comparing the tensile strength of square and reversing half-hitch alternating post knots. Can 7 Surg 2017;60:179-85.

7. Wu V, Yeung C, Sykes EA, et al. Comparison of knot-tying proficiency and knot characteristics for square and reversing half hitch alternating-post surgical knots in a simulated deep body cavity among novice medical students. Can 7 Surg 2018;61:385-91.

8. Schulz KF, Altman DG, Moher D. CONSORT Group. CONSORT 2010 Statement: updated guidelines for reporting parallel group randomized trials. Open Med 2010;4:e60-8.

9. Sheahan G, Reznick R, Klinger D, et al. Comparison of faculty versus structured peer-feedback for acquisitions of basic and intermediate-level surgical skills. Am f Surg 2019;217:214-21.

10. Huang E, Vaughn CJ, Chern H, et al. An objective assessment tool for basic surgical knot-tying skills. F Surg Educ 2015;72:572-6.

11. Ericsson KA. Deliberate practice and acquisition of expert performance: a general overview. Acad Emerg Med 2008;15:988-94.

12. Bann S, Khan M, Datta V, et al. Surgical skill is predicted by the ability to detect errors. Am F Surg 2005;189:412-5.

13. Soucisse ML, Boulva K, Sideris L, et al. Video coaching as an efficient teaching method for surgical residents - a randomized controlled trial. 7 Surg Educ 2017;74:365-71.

14. Thomas MB, Dandolu V, Caputo P, et al. Resident education in principles and technique of bowel surgery using an ex-vivo porcine model. Obstet Gynecol Int 2010;2010:852647.

15. Omotosho P, Yurcisin B, Ceppa E, et al. In vivo assessment of an absorbable and a nonabsorbable knotless barbed suture for laparoscopic single-layer enterotomy closure: a clinical and biomechanical comparison against nonbarbed suture. 7 Laparoendosc Adv Surg Tech A 2011;21:893-7.

16. Demyttenaere SV, Nau P, Henn M, et al. Barbed suture for gastrointestinal closure: a randomized control trial. Surg Innov 2009; 16:237-42.

17. Azari D, Greenberg C, Pugh C, et al. In search of characterizing surgical skill. 7 Surg Educ 2019;76:1348-63.

18. Shandall A, Lowndes R, Young HL. Colonic anastomotic healing and oxygen tension. Br 7 Surg 1985;72:606-9.

19. Palter VN, Grantcharov T, Harvey A, et al. Ex vivo technical skills training transfers to the operating room and enhances cognitive learning: a randomized controlled trial. Ann Surg 2011;253:886-9. 